\title{
Laparoscopic sacrocolpopexy with bone anchor fixation: short-term anatomic and functional results
}

\author{
Mariëlla I. Withagen • Mark E. Vierhout • \\ Guido H. Mannaerts • Robin M. van der Weiden
}

Received: 26 May 2011 / Accepted: 28 October 2011 / Published online: 16 November 2011

(C) The Author(s) 2011. This article is published with open access at Springerlink.com

\begin{abstract}
Introduction and hypothesis The aim of this study was to evaluate short-term anatomic and functional outcomes and safety of laparoscopic sacrocolpopexy with bone anchor fixation.

Methods A prospective cohort study of women undergoing laparoscopic sacrocolpopexy between 2004 and 2009. Anatomic outcome was assessed using the pelvic organ prolapse quantification score (POP-Q). Functional outcomes were assessed using the Urogenital Distress Inventory, Defecatory Distress Inventory, and the Incontinence Impact Questionnaire preoperatively and at 6 months postoperatively. The Wilcoxon signed rank test was used to test differences between related samples.

Results Forty-nine women underwent laparoscopic sacrocolpopexy. The objective success rate in the apical compartment was $98 \%$, subjective success rate was $79 \%$. One mesh exposure (2\%) was found. One conversion was necessary due to injury to the ileum.

Conclusions Laparoscopic sacrocolpopexy with bone anchor fixation is a safe and efficacious treatment for apical
\end{abstract}

M. I. Withagen $(\bowtie) \cdot$ M. E. Vierhout

Department of Obstetrics and Gynecology, Radboud University

Nijmegen Medical Centre,

Postbus 9101, 6500 HB, Nijmegen, The Netherlands

e-mail: m.withagen@obgyn.umcn.nl

R. M. van der Weiden

Department of Obstetrics and Gynecology, Sint Franciscus

Gasthuis,

Rotterdam, The Netherlands

G. H. Mannaerts

Department of Surgery, Sint Franciscus Gasthuis,

Rotterdam, The Netherlands compartment prolapse. It provides excellent apical support and good functional outcome 6 months postoperatively.

Keywords Bone anchor fixation - Functional outcome Laparoscopy $\cdot$ Pelvic organ prolapse $\cdot$ Sacrocolpopexy

\section{Introduction}

A challenging aspect in the treatment of pelvic organ prolapse is the prolapsed apical compartment. The incidence of post-hysterectomy vault prolapse that requires surgery has been estimated at 1.3 per 1,000 women-years [1]. The risk of prolapse surgery was 4.7 times higher in women whose initial hysterectomy was indicated by prolapse and 8.0 times higher if preoperative prolapse stage II or more was present [1].

Numerous surgical procedures have been described for the management of vault prolapse but few have been subject to rigorous assessment of their anatomical and functional outcome. The abdominal sacrocolpopexy is regarded the best procedure compared to vaginal sacrospinous colpopexy in terms of a lower rate of recurrent vault prolapse and less dyspareunia [2]. However, the vaginal sacrospinous colpopexy is quicker and cheaper to perform and women return earlier to activities of daily living. Laparoscopic sacrocolpopexy provides the potential to combine the success rate of an abdominal approach with the faster recovery time associated with a minimally invasive technique. Tissue dissection and mesh placement are facilitated by magnification of the operating field. The success rate of laparoscopic sacrocolpopexy has been reported to be $77-100 \%$ [3-9].

To reduce the risk of injuries to the presacral venous and nervous plexus and to make the procedure more feasible, a 
modified laparoscopic sacrocolpopexy operation using bone anchor fixation and synthetic mesh was developed [10].

The aim of this study was to evaluate the clinical outcomes, surgical morbidity, and functional outcomes of laparoscopic sacrocolpopexy with bone anchor fixation.

\section{Material and methods}

This study was undertaken after obtaining approval from the Central Medical Ethics committee "Toetsing Wetenschappelijk Onderzoek Rijnmond" (TWOR), the Netherlands. All patients provided written informed consent before participation and were recruited between July 2004 and November 2009. Consecutive women with a symptomatic apical compartment prolapse requiring surgical correction were eligible for participation. Exclusion criteria were inability to understand Dutch, pregnancy or contemplating future pregnancy, former rectosigmoid resection, extensive intra-abdominal / pelvic adhesions, body mass index $>40 \mathrm{~kg} / \mathrm{m}^{2}$, and treatment for malignancy in the past.

Baseline evaluation included medical history, a gynecologic investigation including a pelvic organ prolapse quantification (POP-Q)examination [11], and validated urogynecologic questionnaire, which contains the Dutch validated Urogenital Distress Inventory (UDI), Defecatory Distress Inventory (DDI), and the Incontinence Impact Questionnaire (IIQ) [12, 13].

All procedures were performed by the two last authors with the technique as previously described by van der Weiden et al. [10]. The operative procedure can be summarized as follows: at the lowest point of the pouch of Douglas, the right peritoneal fold is opened medially of the rectosigmoid by unipolar diathermy. Next, the presacral avascular plane is developed. The cortical bone of the sacral segment 3 is penetrated in the midline with the laparoscopic bone anchor inserter (SFG prototype, van der Weiden TM or KS 02158-1-LAP TM prototype, Karl Storz, Tuttlingen, Germany). Fixation to the sacrum is performed with a self-tapping titanium Corkscrew Suture Anchor (AR1925S, Arthrex, Naples, Florida, USA) with two attached non-absorbable braided polyester-2 sutures (cases with the SFG prototype, van der Weiden TM) or with flat headed titanium screws (cases with the KS 02158-1-LAP TM prototype, Karl Storz, Tuttlingen, Germany). A $4.0 \times 3.0 \mathrm{~cm}$ piece of monofilament knitted polypropylene mesh (Gynemesh Soft, Ethicon, Norderstedt, Germany) sutured to the apical part of the posterior vaginal wall with four Mersilene 1-0 sutures (Ethicon, Norderstedt, Germany) is subsequently sutured to the polyester-2 ligatures attached to the bone anchor. The mesh is covered with peritoneum placing it in a retroperitoneal position. Since the rate of vaginal mesh exposure is significantly higher when abdominal surgery is combined with vaginal surgery [14], additional prolapse or incontinence procedures such as posterior colpoperineorrhaphy, anterior colporrhaphy, paravaginal repair, surgery for stress urinary incontinence or ventral rectopexy, were not performed concomitantly. Patients received a single prophylactic dose of antibiotics.

Postoperative evaluations were performed during the hospital stay, at 6 weeks and 6 months post-operatively. POP-Q measurements were recorded and questionnaires completed at 6 months.

The primary end point was anatomic failure in any of the vaginal compartments, defined as pelvic organ prolapse stage II or higher. Secondary outcomes were anatomic failure in the apical compartment and subjective failure. Subjective failure was considered significant if a patient responded at least: "yes, a bit bothered" to the questions "Do you feel bulging or protrusion in the vaginal area?" or "Do you see a bulge or protrusion in the vaginal area?" Other secondary outcomes were duration of surgery, blood loss, length of hospitalization, and change in bother and quality of life measured by UDI, DDI, and IIQ. Pain was a secondary outcome as well and considered significant if a patient responded "yes, moderately to quite a bit" to the question "Do you experience pain in the lower abdomen or genital region?" Dyspareunia was considered significant if a patient responded "yes; moderately to quite a bit" to the question "Do you experience pain during intercourse?"

Data are presented as numbers with corresponding percentages or medians with range. The Wilcoxon signed rank test was used to test differences between related samples for statistical significance. A $p$ value below 0.05 was considered statistically significant. Statistical analysis was performed using SPSS 18.0 for Windows (SPSS Inc., Chicago, Ill., USA).

\section{Results}

Forty-nine women were included in the study, 45 cases of laparoscopic sacrocolpopexy (including two cases of cervicopexy after a previous supracervical abdominal hysterectomy) and four cases of laparoscopic hysteropexy. All patients returned for follow-up and a POP-Q measurement was taken; however, only 47 patients completed the questionnaires. Baseline characteristics are shown in Table 1. Two patients had a bothersome stage I prolapse with the most descending point at hymen minus $2 \mathrm{~cm}$ and underwent surgery. All other patients had a stage II or III prolapse. Twenty patients were sexually active and three $(15 \%)$ had dyspareunia.

Peri- and postoperative data are presented in Table 2. In one patient, conversion was necessary due to injury to the 
Table 1 Baseline characteristics

Data available $N(\%)$ or median for number of (range) patients

\begin{tabular}{lcc}
\hline Age & 49 & $66(45-86)$ \\
Parity & 47 & $2(0-6)$ \\
BMI & 29 & $24(21-32)$ \\
Comorbidity & 49 & $5(10 \%)$ \\
Previous surgery & \\
Abdominal hysterectomy & 49 & $18(37 \%)$ \\
Vaginal hysterectomy & 49 & $27(55 \%)$ \\
Anterior colporraphy & 49 & $28(57 \%)$ \\
Anterior mesh procedure & 49 & $1(2 \%)$ \\
Posterior colporrhaphy & 49 & $27(55 \%)$ \\
One POP procedure & 49 & $28(57 \%)$ \\
More than one POP procedure & 49 & $5(10 \%)$ \\
Previous incontinence surgery & 49 & $9(18 \%)$ \\
Overall POP-stage & 49 & \\
I & & $2(4 \%)$ \\
II & & $33(67 \%)$ \\
III & & $14(29 \%)$ \\
IV & & $0(0 \%)$ \\
Point C & 49 & $-1(-3$ to 4$)$ \\
Pain (lower abdomen/genital area) & 47 & $11(23 \%)$ \\
Dyspareunia & 20 & $3(15 \%)$ \\
\hline
\end{tabular}

${ }^{\mathrm{a}}$ Multiple surgeries possible

Table 2 Peri- and post-operative data

\begin{tabular}{lcc}
\hline & $\begin{array}{l}\text { Data available } \\
\text { for number of } \\
\text { patients }\end{array}$ & \\
& (range)
\end{tabular}

ileum. The lesion was sutured and subsequently healed without any long-term sequelae. One patient had neurologic complaints post-operatively, caused by irritation of the left sacral plexus. These complaints, although diminishing gradually, persisted to some extent at the 6 months follow-up and resolved spontaneously in the following 6 months. One patient developed a mesh exposure, which was successfully excised and the defects covered with vaginal mucosa in a day-care procedure. There were no patients that developed de novo pain in the abdomen or genital area and three patients $(15 \%)$ reported de novo dyspareunia. In two patients $(10 \%)$, dyspareunia disappeared.

The POP-Q measurements are shown in Table 3. At 6 months follow-up, a significant improvement was seen in the apical (point $\mathrm{C}$ ) and posterior (point $\mathrm{Bp}$ ) compartments. Anatomical failure per compartment is presented in Table 4. The apical compartment had only $2 \%$ failures, in contrast with the anterior compartment with $31 \%$ failures. Subjective failure was demonstrated in 10 out of 47 (21\%) patients. Of the 26 patients with an overall anatomic failure, only 7 patients (27\%) had bothersome prolapse complaints (subjective failure). Of the 23 patients with an overall POP stage 0 or I, three patients $(13 \%)$ had bothersome prolapse complaints (subjective failure). Changes in anterior (point $\mathrm{Ba}$ ), apical (point $\mathrm{C}$ ), and posterior (point $\mathrm{Bp}$ ) compartments are shown in Table 5 . Improvement in the apical compartment ranged from 5 to $12 \mathrm{~cm}$ and was present in all cases but one. The majority of patients had no change in the anterior and posterior compartment.

At 6 months, the bother scores of genital prolapse, obstructive micturition, overactive bladder, pain, and obstructed defecation were significant improved (Table 6). Bother scores were higher when the POP stage increased, although this was not statistically significant.

\section{Discussion}

The data in this study show that that laparoscopic sacrocolpopexy with bone anchor fixation is a safe and effective surgical treatment for apical compartment prolapse. The procedure provided excellent vault support in 48 out of 49 women $(98 \%)$. This high success rate for the apical compartment is comparable with other studies [4-9].

Subjective success was $79 \%$, comparable with numbers reported by Higgs et al. [8], and just below the previously reported subjective success rates of $86 \%$ to $97 \%[4,9]$. This lower rate of subjective success was probably due to the persistent prolapse of the anterior or posterior vaginal wall that was found in 26 women (53\%). This results in a high number of overall failures. A cause for this high overall 
Table 3 POP-Q measurements at baseline and 6 months post-surgery

\begin{tabular}{|c|c|c|c|c|c|c|}
\hline \multirow[t]{2}{*}{ POP-Q point } & \multirow{2}{*}{$\begin{array}{l}\text { Data available for } \\
\text { number of patients }\end{array}$} & \multicolumn{2}{|l|}{ Baseline } & \multicolumn{2}{|l|}{6 months } & \multirow[t]{2}{*}{$p$ value } \\
\hline & & Median (range) & Mean (SD) & Median (range) & Mean (SD) & \\
\hline $\mathrm{Aa}$ & 49 & $-2(-3$ to 1$)$ & $-1.3(1.0)$ & $-2(-3$ to 0$)$ & $-1.7(0.7)$ & 0.007 \\
\hline $\mathrm{Ba}$ & 49 & $-2(-3$ to 3$)$ & $-0.9(1.6)$ & $-2(-3$ to 2$)$ & $-1.4(1.2)$ & 0.003 \\
\hline $\mathrm{C}$ & 49 & $-1(-3$ to 4$)$ & $-0.1(1.8)$ & $-8(-8$ to -3$)$ & $-7.7(0.9)$ & $<0.001$ \\
\hline GH & 49 & $3(2$ to 5$)$ & $3.0(0.7)$ & $3(2$ to 5$)$ & $3.0(0.7)$ & 0.99 \\
\hline $\mathrm{PB}$ & 49 & $3(2$ to 3$)$ & $2.7(0.5)$ & $3(2$ to 3$)$ & $3.0(0.5)$ & 0.16 \\
\hline TVL & 49 & $8(7$ to 9$)$ & $8.0(0.2)$ & 8 (7 to 9$)$ & $8.0(0.2)$ & 0.99 \\
\hline Ap & 49 & $-2(-3$ to 2$)$ & $-1.4(0.9)$ & $-2(-3$ to 0$)$ & $-1.9(0.5)$ & $<0.001$ \\
\hline $\mathrm{Bp}$ & 49 & -1 ( -3 to 2$)$ & $-0.9(1.4)$ & $-2(-3$ to 2$)$ & $-1.6(0.9)$ & $<0.001$ \\
\hline $\mathrm{D}$ & 4 & $-2.5(-4$ to 0$)$ & $-2.3(1.7)$ & $-8(-8$ to -5$)$ & $-7.3(1.5)$ & 0.07 \\
\hline
\end{tabular}

failure rate could be the fact that we did not combine the apical compartment repair with anterior or posterior repairs (either with laparoscopic mesh placement or with vaginal colporrhaphia). In order to decrease the risk for mesh exposure [14] and to avoid irritable bladder symptoms, such as de novo urgency of $18 \%$ [15], and to avoid rectal or bladder lesions we did not perform combined repairs. Theoretically, the fixation of the mesh to sacral segment 2 to 4 , instead of the promontorium, could prevent the occurrence or recurrence of prolapse, since the axis of the vagina is more natural and further support is provided by the levator plate against which the upper vagina is compressed by intra-abdominal pressure [16]. Furthermore, adequate level I support has a critical role on the position of the anterior and posterior vagina $[17,18]$. However, this adequate level I support has not prevented the occurrence of anterior and posterior wall prolapse in all cases.

For the definition of failure, we used the National Institutes of Health $(\mathrm{NIH})$ criteria [11, 19]. However, according to Barber, the presence of "bulge" symptoms together with a pelvic organ prolapse beyond the hymen or re-treatment would be more appropriate. If we look at our data in this way, we have a failure rate of 6 out of $49(12 \%)$ [20].

Intraoperative complications were rare. Suspending the vault to the sacral segment 2-4 with a bone anchor avoids large difficult promontory dissection, declining the risk of presacral hemorrhage which can be difficult to control resulting in conversion to laparotomy [21]. Blood loss during surgery was lower (median $50 \mathrm{ml}(10-100 \mathrm{ml})$, compared to $100 \mathrm{ml}$ (range $20-300 \mathrm{ml}$ ) in a previous study [3]. Furthermore, no blood transfusion peri-operatively was given, in contrast with $2 \%$ transfusions in the study of Maher et al. [3]. Neither bladder lesions nor rectum lesions were described, due to the minimum dissection at the bladder and rectum site, since mesh was only used at the apex of the posterior vaginal wall. Other studies described $2 \%$ bladder lesions and $1 \%$ rectum lesions [7, 9]. However, one conversion to laparotomy was necessary due to injury to the ileum in a patient with extensive adhesion formation.

Table 4 Anatomic failure

\begin{tabular}{|c|c|c|c|c|c|c|c|c|}
\hline \multirow[t]{2}{*}{ POP-stage } & \multicolumn{2}{|c|}{ Anterior compartment } & \multicolumn{2}{|c|}{ Apical compartment } & \multicolumn{2}{|c|}{ Posterior compartment } & \multicolumn{2}{|l|}{ Overall } \\
\hline & Baseline & 6 months & Baseline & 6 months & Baseline & 6 months & Baseline & 6 months \\
\hline Stage 0 & $2(4 \%)$ & $3(6 \%)$ & $2(4 \%)$ & $49(100 \%)^{\mathrm{a}}$ & $1(2 \%)$ & $3(6 \%)$ & $0(0 \%)$ & $2(4 \%)$ \\
\hline Stage I & $25(51 \%)$ & $31(63 \%)$ & $9(19 \%)$ & $0(0 \%)$ & $23(47 \%)$ & $34(69 \%)$ & $2(4 \%)$ & $21(43 \%)$ \\
\hline Stage II & $17(35 \%)$ & $12(24 \%)$ & $30(61 \%)$ & $0(0 \%)$ & $21(43 \%)$ & $11(22 \%)$ & $33(67 \%)$ & $22(45 \%)$ \\
\hline Stage III & $5(10 \%)$ & $3(6 \%)$ & $8(16 \%)$ & $0(0 \%)$ & $4(8 \%)$ & $1(2 \%)$ & $14(29 \%)$ & $4(8 \%)$ \\
\hline Stage IV & $0(0 \%)$ & $0(0 \%)$ & $0(0 \%)$ & $0(0 \%)$ & $0(0 \%)$ & $0(0 \%)$ & $0(0 \%)$ & $0(0 \%)$ \\
\hline Failure & & $15(31 \%)$ & & $1(2 \%)$ & & $12(24 \%)$ & & $26(53 \%)$ \\
\hline
\end{tabular}

Data presented as numbers $(\%)$

Failure $=\mathrm{POP} \geq$ stage II or $\mathrm{POP}<$ stage II with repeat surgery

${ }^{a}$ Including one repeat sacrocolpopexy within 6 months 
Table 5 Change in POP-Q point $\mathrm{Ba}, \mathrm{C}$, and $\mathrm{Bp} 6$ months postsurgery

\begin{tabular}{llll}
\hline & $\mathrm{Ba}$ & $\mathrm{C}$ & $\mathrm{Bp}$ \\
\hline $\begin{array}{lll}\text { Improvement } \\
1 \mathrm{~cm}\end{array}$ & $9(19 \%)$ & & $10(21 \%)$ \\
$2 \mathrm{~cm}$ & $3(6 \%)$ & & $3(6 \%)$ \\
$3 \mathrm{~cm}$ & $4(8 \%)$ & & $8(16 \%)$ \\
$4 \mathrm{~cm}$ & $1(2 \%)$ & & \\
$5 \mathrm{~cm}$ & & $4(8 \%)$ & \\
$6 \mathrm{~cm}$ & & $10(20 \%)$ & \\
$7 \mathrm{~cm}$ & & $13(27 \%)$ & \\
$8 \mathrm{~cm}$ & & $5(10 \%)$ & \\
$9 \mathrm{~cm}$ & & $1(2 \%)$ & \\
$10 \mathrm{~cm}$ & & $2(4 \%)$ & \\
$11 \mathrm{~cm}$ & & 0 & \\
$12 \mathrm{~cm}$ & & & \\
No change & $28(57 \%)$ & 0 & \\
Deterioration & & 0 & \\
$1 \mathrm{~cm}$ & $2(4 \%)$ & $1(2 \%)$ & \\
$2 \mathrm{~cm}$ & $2(4 \%)$ & & \\
Repeat surgery & & & \\
\hline
\end{tabular}

Medium-term complications included one case (2\%) of mesh exposure. This finding is low to consistent with the $1-9 \%$ rate of mesh exposure previously reported for sacrocolpopexy by either the open or the laparoscopic route [3, 4, 6-9]. De novo pain was not found, compared to $4 \%$ in a group with vaginal native tissue repair, and $7.5 \%$ in a group with vaginal mesh repair previously described [22]. De novo dyspareunia rate of $15 \%$ is comparable to rates of $8-42 \%$ that have been reported in the literature after conventional vaginal prolapse surgery and after mesh repair [22-25].

The short follow-up of 6 months was an important limitation of this study. Furthermore, an independent clinical investigator who was not involved in the procedures would ideally have performed the postoperative examinations. The strengths of this study are the prospective design of the study, the uniform surgical technique performed by the same gynecologist and laparoscopic surgeon, and the use of standardized and validated instruments of measurement.

\section{Conclusions}

Laparoscopic sacrocolpopexy with bone anchor fixation is a safe surgical treatment and has a high short-term success rate for apical compartment prolapse. For anterior and/or posterior compartment prolapse in the same patient, additional surgery (with laparoscopic mesh or vaginal colporrhaphy) should be considered.

Table 6 Effect of surgery on symptoms and health related quality of life scores

\begin{tabular}{|c|c|c|c|c|c|c|}
\hline \multirow[t]{2}{*}{ Domain } & \multirow[t]{2}{*}{ Baseline } & \multirow[t]{2}{*}{6 months } & \multirow[t]{2}{*}{$p$ value } & \multicolumn{3}{|c|}{ Bother score in relation to POP stage at 6 months } \\
\hline & & & & POP stage $0+\mathrm{I}(n=21)$ & POP stage II $(n=20)$ & POP stage III $(n=4)$ \\
\hline \multicolumn{7}{|l|}{ UDI } \\
\hline Genital prolapse & $67.8(28.8)$ & $13.3(24.5)$ & $<0.001$ & $10.3(23.3)$ & $13.3(24.5)$ & $33.3(33.3)$ \\
\hline $\mathrm{OAB}$ & $33.3(25.7)$ & $17.3(18.9)$ & $<0.001$ & $12.7(14.2)$ & $16.1(17.8)$ & $47.2(22.9)$ \\
\hline Incontinence & $15.6(20.1)$ & $10.0(16.8)$ & 0.09 & $7.9(17.2)$ & $10.0(16.6)$ & $20.8(16.0)$ \\
\hline Obstructive micturition & $33.0(24.7)$ & $14.8(21.3)$ & $<0.001$ & $11.9(16.8)$ & $13.3(20.7)$ & $44.4(38.5)$ \\
\hline Pain & $26.8(25.2)$ & $15.2(19.3)$ & 0.002 & $11.1(16.1)$ & $18.3(22.2)$ & $22.2(19.2)$ \\
\hline \multicolumn{7}{|l|}{ DDI } \\
\hline Constipation & $13.0(19.5)$ & $12.4(20.4)$ & 0.56 & $5.6(13.3)$ & $17.5(23.9)$ & $27.8(25.5)$ \\
\hline Obstructed defecation & $13.5(14.4)$ & $7.7(11.1)$ & 0.002 & $6.3(12.3)$ & $7.9(9.4)$ & $20.8(5.9)$ \\
\hline Pain & $6.7(15.8)$ & $7.0(15.5)$ & 0.84 & $4.8(15.0)$ & $8.8(16.1)$ & $11.1(19.3)$ \\
\hline Incontinence & $4.0(11.2)$ & $4.5(9.8)$ & 1.00 & $5.6(12.2)$ & $2.5(6.1)$ & $11.1(9.6)$ \\
\hline \multicolumn{7}{|l|}{ IIQ } \\
\hline Physical functioning & $19.8(26.3)$ & $13.6(23.4)$ & 0.14 & $15.0(23.5)$ & $11.4(24.9)$ & $16.7(19.3)$ \\
\hline Mobility & $25.5(20.5)$ & $23.5(24.8)$ & 0.53 & $23.9(23.7)$ & $21.1(26.7)$ & $33.3(24.0)$ \\
\hline Social functioning & $12.8(11.6)$ & $7.9(13.6)$ & 0.08 & $8.8(16.0)$ & $7.1(11.7)$ & $5.6(7.9)$ \\
\hline Embarrassment & $11.5(20.0)$ & $6.5(13.4)$ & 0.15 & $6.7(13.7)$ & $4.6(9.6)$ & $16.7(28.9)$ \\
\hline Emotional health & $22.2(19.8)$ & $16.1(21.9)$ & 0.17 & $17.8(22.9)$ & $14.2(22.5)$ & $16.7(7.9)$ \\
\hline
\end{tabular}

UDI, DDI, IIQ data presented as mean (standard deviation)

Scores range between 0 (least bother) and 100 (maximum bother) 
Acknowledgements None.

Funding None.

Conflict of interest R.M.F. van der Weiden developed the Franciscan screw driver for the bone anchor insertion and is patent holder (together with KARL STORZ GmbH, Tuttlingen, Germany).

Open Access This article is distributed under the terms of the Creative Commons Attribution Noncommercial License which permits any noncommercial use, distribution, and reproduction in any medium, provided the original author(s) and source are credited.

\section{References}

1. Dallenbach P, Kaelin-Gambirasio I, Dubuisson JB, Boulvain M (2007) Risk factors for pelvic organ prolapse repair after hysterectomy. Obstet Gynecol 110:625-632

2. Maher C, Feiner B, Baessler K et al (2010) Surgical management of pelvic organ prolapse in women. Cochrane Database Syst Rev 4:CD004014

3. Maher CF, Feiner B, DeCuyper EM et al (2011) Laparoscopic sacral colpopexy versus total vaginal mesh for vaginal vault prolapse: a randomized trial. Am J Obstet Gynecol 204(4):360367

4. Agarwala N, Hasiak N, Shade M (2007) Laparoscopic sacral colpopexy with Gynemesh as graft material: experience and results. J Minim Invasive Gynecol 14:577-583

5. Sundaram CP, Venkatesh R, Landman J, Klutke CG (2004) Laparoscopic sacrocolpopexy for the correction of vaginal vault prolapse. J Endourol 18:620-623

6. Ross JW, Preston M (2005) Laparoscopic sacrocolpopexy for severe vaginal vault prolapse: 5-year outcome. J Minim Invasive Gynecol 12:221-226

7. Cosson M, Rajabally R, Bogaert E et al (2002) Laparoscopic sacrocolpopexy, hysterectomy, and Burch colposuspension: feasibility and short-term complications of 77 procedures. JSLS 6:115-119

8. Higgs PJ, Chua HL, Smith AR (2005) Long term review of laparoscopic sacrocolpopexy. BJOG 112:1134-1138

9. Price N, Slack A, Jackson SR (2010) Laparoscopic sacrocolpopexy: an observational study of functional and anatomical outcomes. Int Urogynecol J Pelvic Floor Dysfunct 22(1):77-82

10. van der Weiden RM, Withagen MI, Bergkamp AB, Mannaerts GH (2005) A new device for bone anchor fixation in laparoscopic sacrocolpopexy. The Franciscan laparoscopic bone anchor inserter. Surg Endosc 19:594-597

11. Bump RC, Mattiasson A, Bo K et al (1996) The standardization of terminology of female pelvic organ prolapse and pelvic floor dysfunction. Am J Obstet Gynecol 175:10-17

12. Van der Vaart CH, de Leeuw JR, Roovers JP, Heintz AP (2003) Measuring health-related quality of life in women with urogenital dysfunction: the urogenital distress inventory and incontinence impact questionnaire revisited. Neurourol Urodyn 22:97-104

13. Brummen HJ, Bruinse HW, Pol G, Heintz AP, Vaart CH (2006) Defecatory symptoms during and after the first pregnancy: prevalences and associated factors. Int Urogynecol J Pelvic Floor Dysfunct 17:224-230

14. Visco AG, Weidner AC, Barber MD et al (2001) Vaginal mesh erosion after abdominal sacral colpopexy. Am J Obstet Gynecol 184:297-302

15. Patel M, O’Sullivan D, Tulikangas PK (2009) Is Burch or midurethral sling better with abdominal sacral colpopexy? Int Urogynecol J 20:787-790

16. Nichols DH, Milley PS, Randall CL (1970) Significance of restoration of normal vaginal depth and axis. Obstet Gynecol 2:251-256

17. DeLancey JOL (1992) Anatomic aspects of vaginal eversion after hysterectomy. Am J Obstet Gynecol 166:1717-1724

18. Lowder JL, ParkAJ ER et al (2008) The role of apical vaginal support in the appearance of anterior and posterior vaginal prolapse. Obstet Gynecol 111:152-157

19. Weber AM, Abrams P, Brubaker L et al (2001) The standardization of terminology for researchers in female pelvic floor disorders. Int Urogynecol J Pelvic Floor Dysfunct 12:178-186

20. Barber MD, Brubaker L, Nygaard I et al (2009) Defining success after surgery for pelvic organ prolapse. Obstet Gynecol 114:600-609

21. Wattiez A, Canis M, Mage G et al (2001) Promontofixation for the treatment of prolapse. Urol Clin N Am 28:151-157

22. Withagen MI, Milani AL, Boon J, Vervest HA, Vierhout ME (2011) Trocar-guided mesh versus conventional vaginal repair in recurrent prolapse; randomized controlled trial. Obstet Gynecol 117:242-250

23. Sergent F, Zanati J, Bisson V, Desilles N, Resch B, Marpeau L (2010) Perioperative course and medium-term outcome of the transobturator and infracoccygeal hammock for posthysterectomy vaginal vault prolapse. Int J Gynaecol Obstet 109:131-135

24. Lowman JK, Jones LA, Woodman PJ, Hale DS (2008) Does the prolift system cause dyspareunia? Am J Obstet Gynecol 199(707): e1-e6

25. Carey M, Higgs P, Goh J et al (2009) Vaginal repair with mesh versus colporrhaphy for prolapse: a randomised controlled trial. BJOG 116:1380-1386 